\title{
La promoción y gestión de los polígonos industriales en la Eurorregión Galicia-Norte de Portugal
}

\author{
Jesús García-Arca, ${ }^{\mathrm{a}, *}$, José Antonio Comesaña-Benavides ${ }^{\mathrm{b}}$, \\ José Carlos Prado-Prado ${ }^{c}$ \\ a,*jgarca@uvigo.es, Universidad de Vigo, Espanha

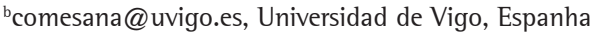 \\ cjcprado@uvigo.es, Universidad de Vigo, Espanha
}

\section{Resumen}

En un entorno global, para reforzar la competitividad de las empresas es necesario adoptar políticas de desarrollo de infraestructuras logísticas y de polígonos industriales. Sin embargo, a pesar del impacto económico y social que generan estos polígonos industriales, su adecuada promoción y gestión no se ha destacado, especialmente en España. En este contexto, este artículo profundiza en las causas que conducen a una deficiente promoción y gestión de los polígonos industriales, proponiendo líneas de actuación su mejora. Para ilustrar esta problemática general, se analiza la promoción y gestión de los polígonos industriales en la Eurorregión Galicia-Norte de Portugal (provincia de Pontevedra en España y la zona de Minho-Lima en el distrito portugués de Viana do Castelo). Si bien el estudio de campo se centra en el ámbito fronterizo de la Eurorregión Galicia-Norte de Portugal, las conclusiones y recomendaciones del estudio son perfectamente extrapolables al resto de España y Portugal.

Palabras clave

Polígonos industriales. Promoción. Gestión.

\section{Introducción}

En un entorno económico global, cada vez más sectores productivos se ven sometidos a la competencia de las economías emergentes y a la amenaza de la deslocalización. Afrontar con visión estratégica esta realidad requiere la adopción de medidas integradas con objeto de facilitar las actividades desarrolladas, no sólo para las empresas ya ubicadas en un área geográfica sino, también, para las potenciales empresas que pudieran contemplar esta ubicación en el futuro. En este contexto, para reforzar la competitividad de las empresas existentes y potenciales es necesaria la adopción de políticas de desarrollo de infraestructuras logísticas y polígonos industriales* (CHRISTOPHER; TOWILL, 2002; KEARNEY, 2009).

*Con el concepto de polígono industrial o parque industrial, realmente, se pueden englobar cuatro grandes categorías: los parques científicos o tecnológicos, los parques especializados, los parques multifuncionales (los más habituales) y los logísticos. En este artículo se hará referencia indistintamente a todos ellos con el término general de polígonos industriales.
Ahora bien, para que estos polígonos industriales puedan materializarse es necesario que entren en escena, al menos, dos entes: el promotor y el gestor.

Así, se entiende por "promotor" toda aquella empresa, organización o institución que promueve una actuación urbanística (con la presentación de un plan urbanístico o aceptando la ejecución del mismo) asumiendo frente a la Administración Urbanística la realización del plan, previo cumplimiento de las obligaciones de urbanización, cesión y, en su caso, equidistribución (Cooperación, Compensación,...). El Plan Urbanístico es la norma que rige todas las actuaciones, sometiéndose a él tanto los entes privados como los públicos. Estos planes urbanísticos se articulan con los Planes Generales de Ordenación Urbana (PGOU).

Existe la posibilidad (aunque raramente empleada) de que se elaboren PGOU conjuntos, o Planes Comarcales que afecten a varios municipios. 
Como lamentablemente no siempre existe un PGOU operativo, se hadesarrollado este planeamiento aplicando el concepto del proyecto sectorial (con incidencia "supramunicipal" que pueden ser desarrollados por iniciativa pública o privada, con autorización autonómica).

Por otro lado, el gestor de polígonos industriales es una entidad pública, privada o mixta que planifica una serie de actividades para lograr, tanto un funcionamiento adecuado de las infraestructuras existentes, como para la creación y mantenimiento de servicios necesarios para las empresas. El mejor momento para decidir la forma jurídica de la entidad gestora de un polígono es durante la fase de diseño y planeamiento dado que se podrían definir los servicios para los compradores de suelo, así como sus responsabilidades. A posteriori, es difícil crearla salvo que exista la unanimidad de los propietarios de las parcelas.

No obstante, la conservación básica de los polígonos industriales corresponde a los ayuntamientos (excepto en el caso de urbanizaciones privadas), si bien limitada por ley a ciertas obras básicas (Cuadro 1), la modalidad de gestión exclusiva del ayuntamiento es muy habitual en los polígonos activos.La legislación contempla dos alternativas de gestión complementarias a la del ayuntamiento: la entidad de conservación y la comunidad de propietarios.

En este escenario, surge como dinamizador en la gestión de los polígonos y de las ZIEs (Zonas de Industrialización Espontánea que por sus deficiencias no pueden considerarse polígonos), las asociaciones de empresarios, actuando como mediadores entre las administraciones y las empresas, promoviendo, asimismo, la puesta en marcha de mejoras de infraestructuras y servicios. En lo Cuadro 1, se presentan las principales ventajas e inconvenientes de cada una de las figuras comentadas.

A pesar del impacto económico y social que generan los polígonos industriales (en Galicia dan cabida, al menos, a más de 3.400 empresas y más de 60.000 trabajadores; (FEGAPE, 2008)), la promoción y la gestión de los polígonos en España no es especialmente positiva. Así, en un estudio de Mora (2006) se muestra que más del 50\% de los polígonos carecen de la red mínima contra incendios, que el 20\% carecen de alumbrado público y que sólo el 1\% dispone de servicio de guardería, existiendo, incluso, polígonos con calles sin asfaltar y sin servicio de agua.

Más aún, en este mismo estudio se muestra que el $75 \%$ de los polígonos españoles no contaban con una entidad específica para realizar la gestión de los polígonos industriales (más allá de los ayuntamientos), llegando al 88\% en los polígonos de la Comunidad Valenciana (la segunda comunidad en suelo industrial, tras Andalucía).

\section{Objetivos y metodología del estudio}

En este contexto, este artículo profundiza en las causas que conducen a una deficiente promoción y gestión de los polígonos industriales, proponiendo líneas de actuación para mejorar la situación actual. Para ilustrar esta problemática general, se analiza la situación en la promoción y gestión de polígonos en la Eurorregión Galicia-Norte de Portugal, más

Cuadro 1. Ventajas e inconvenientes de los diferentes agentes que participan en la gestión de los polígonos (Adaptado de Mora (2006) y FEPEVAL, (2006)).

\begin{tabular}{|c|c|c|}
\hline & Ventajas & Inconvenientes \\
\hline Ayuntamiento & $\begin{array}{l}\text { - Obligación legal de mantenimiento de infraestructuras } \\
\text { y servicios mínimos (obras de vialidad, saneamiento, } \\
\text { alcantarillado, suministro de agua potable, suministro } \\
\text { de energía eléctrica y jardineria) }\end{array}$ & $\begin{array}{l}\text { - Cierta dejadez en sus responsabilidades } \\
\text { - ¿Recursos insuficientes? (al menos, así lo denuncian los } \\
\text { ayuntamientos) }\end{array}$ \\
\hline $\begin{array}{l}\text { Entidad de } \\
\text { conservación }\end{array}$ & $\begin{array}{l}\text { - Obligación de asociarse a priori (evita efecto "parásito" } \\
\text { de los no asociados) } \\
\text { - Vía de apremio para el cobro de cuotas } \\
\text { - Implicación del Ayuntamiento en la gestión }\end{array}$ & $\begin{array}{l}\text { - Integra a los propietarios y no a los empresarios } \\
\text { - Limitación en la prestación de servicios } \\
\text { - Incapacidad para presentarse a subvenciones } \\
\text { - Elevados costes (en ocasiones duplicados) }\end{array}$ \\
\hline $\begin{array}{c}\text { Comunidad de } \\
\text { propietarios }\end{array}$ & $\begin{array}{l}\text { - Evitan la tutela del Ayuntamiento } \\
\text { - Cobro de cuotas } \\
\text { - Menores tasas municipales } \\
\text { - Explotación de zonas comunes }\end{array}$ & $\begin{array}{l}\text { - Falta de personalidad jurídica } \\
\text { - Incapacidad para presentarse a subvenciones } \\
\text { - Integra a los propietarios y no a los empresarios } \\
\text { - Si privado, Ayuntamiento no entra } \\
\text { - Limitación en la prestación de servicios }\end{array}$ \\
\hline $\begin{array}{l}\text { Asociación de } \\
\text { empresarios }\end{array}$ & $\begin{array}{l}\text { - Carácter voluntario: convencimiento de los asociados } \\
\text { (usuarios) y proactividad } \\
\text { - Libertad para la prestación y desarrollo de servicios } \\
\text { - Entidad sin ánimo de lucro } \\
\text { - Acceso a subvenciones }\end{array}$ & $\begin{array}{l}\text { - Carácter voluntario: problemas de asociación y financiación } \\
\text { - Posible choque de competencias con otras entidades de } \\
\text { carácter sectorial } \\
\text { - Limitaciones en las relaciones con la Administración }\end{array}$ \\
\hline
\end{tabular}


concretamente centrada en el área de Pontevedra (España) y la zona de Minho-Lima (distrito de Viana do Castelo en Portugal).

Este trabajo es fruto de un estudio realizado por los autores para la Confederación de Empresarios de Pontevedra (CEP) en el que se ha realizado una profunda revisión bibliográfica sobre la promoción y gestión de los polígonos industriales a nivel de España y Portugal; dicha revisión ha servido, no sólo para fundamentar las recomendaciones que se proponen sino, también, para realizar el diseño de los cuestionarios/guiones empleados en la posterior etapa del estudio de campo (que ha servido para enriquecer, complementar y matizar estas recomendaciones).

En la etapa del estudio de campo se ha intentado captar, de primera mano, las necesidades y las opiniones de los diferentes colectivos relacionados o afectados por la promoción y gestión de polígonos industriales. Para ello, se ha dividido esta fase en tres análisis complementarios, tal como se puede observar en la Tabla 1.

En este artículo se comentarán especialmente los aspectos cuantitativos valorados por las empresas pontevedresas, si bien, se ilustrará en aspectos específicos con datos proporcionados por los otros colectivos estudiados. En este contexto, si bien, el estudio de campo se centra en el ámbito fronterizo de la Eurorregión Galicia-Norte de Portugal, las conclusiones y recomendaciones del estudio son extrapolables al resto de España.

\section{Resultados del estudio de campo}

Gracias a la información recabada de las fuentes bibliográficas revisadas (GARCÍA-CEBRIÁN; MUÑOZ, 2009) y a las entrevistas realizadas ha sido posible caracterizar, comparativamente, las principales variables relacionadas con la promoción y la gestión de los polígonos en Pontevedra y Norte de Portugal, las cuales se sintetizan en lo Cuadro 2.

Tras esta etapa de caracterización previa de la situación de partida, se ha profundizado en el análisis de las respuestas de las empresas ubicadas en los polígonos industriales de Pontevedra. Así, en primer lugar, se ha preguntado a las empresas por los motivos que les han llevado a elegir su ubicación actual.

En la Figura 1 se muestran los resultados obtenidos en los cinco motivos más importantes. Como puede observarse en el gráfico, el principal motivo por el que las empresas se ubican en los polígonos es la disponibilidad de espacio (además, es el motivo más citado tanto en primer lugar como en segundo y tercer lugar, mencionado por el 76\% de las empresas), siendo el segundo motivo, por orden de importancia, el precio. Complementando estos dos motivos, la aparición de una oportunidad interesante en relación con espacio adecuado a buen precio. Otros dos factores importantes son la cercanía del polígono a los clientes más importantes de la empresa y las buenas comunicaciones del polígono.

De forma similar a la pregunta anterior, en este apartado se ha preguntado a las empresas por los principales inconvenientes que presentaba la ubicación actual. En la Figura 2 se muestran los resultados obtenidos en los cinco motivos más importantes. Así, se observa que el precio elevado es el principal inconveniente citado por las empresas, seguido de las comunicaciones deficientes del polígono. La falta de espacio, demasiado justo o sin posibilidad de crecer en el futuro ocupa el tercer lugar. Completan este resumen de los 5 principales inconvenientes la falta de ayuda para implantarse 0 la distancia excesiva a los clientes.

Tabla 1. Ficha técnica del estudio de campo.

\begin{tabular}{|c|c|c|c|}
\hline & $\begin{array}{l}\text { Empresas ubicadas } \\
\text { en los } 30 \text { polígonos } \\
\text { industriales de } \\
\text { Pontevedra }\end{array}$ & $\begin{array}{l}\text { Empresas españolas } \\
\text { ubicadas en la zona } \\
\text { limitrofe del Norte de } \\
\text { Portugal }\end{array}$ & $\begin{array}{l}\text { Entidades relacionadas con la promoción y gestión de } \\
\text { polígonos industriales en Pontevedra y Norte de Portugal }\end{array}$ \\
\hline Universo & $\begin{array}{l}559 \\
\text { (fuente SAB1 2007) }\end{array}$ & $\begin{array}{l}22 \text { empresas } \\
\text { (estimación propias a } \\
\text { partir SAB1 2007) }\end{array}$ & $\begin{array}{l}\text { - } 12 \text { Asociaciones de Empresarios en polígonos industriales } \\
\text { de Pontevedra; } \\
\text { - Promotores, gestores y otras entidades relacionadas. }\end{array}$ \\
\hline $\begin{array}{l}\text { Técnica } \\
\text { empleada }\end{array}$ & $\begin{array}{l}\text { Entrevistas personales } \\
\text { y envios postales con } \\
\text { cuestionario estructurado }\end{array}$ & $\begin{array}{l}\text { Entrevistas personales con } \\
\text { cuestionario estructurado }\end{array}$ & - Entrevistas personales con cuestionario estructurado. \\
\hline $\begin{array}{l}\text { Cuestionarios } \\
\text { válidos }\end{array}$ & $51(9,12 \%)$ & $4(18,18 \%)$ & $\begin{array}{l}\text { - } 8 \text { Asociaciones de Empresarios de Polígonos (67\%); } \\
\text { - } 3 \text { promotores de suelo industrial (los } 2 \text { principales de } \\
\text { Pontevedra y el principal del Norte de Portugal); } \\
\text { - FEGAPE (Federación Gallega de Polígonos Empresariales) y } \\
\text { - } 1 \text { asesor inmobiliario de suelo industrial en Galicia. }\end{array}$ \\
\hline
\end{tabular}


En otro aspecto, se valoró la situación logística del polígono en cuatro aspectos: los accesos por carreteras de alta capacidad, la proximidad a aeropuertos, terminales de carga de ferrocarril y puertos, tanto a nivel de interés o importancia para su negocio como a nivel de satisfacción con

Cuadro 2. Comparativa de la situación de la promoción y gestión de polígonos industriales en Pontevedra y el Norte de Portugal (elaboración propia).

\begin{tabular}{|c|c|c|}
\hline & Pontevedra & Zona del Norte de Portugal estudiada \\
\hline $\begin{array}{l}\text { Polígonos } \\
\text { Industriales en } \\
\text { funcionamiento }\end{array}$ & $\begin{array}{l}30 \text { polígonos industriales con casi } 12 \text { millones de } \mathrm{m}^{2} \text {; } \\
\text { además, se podrían añadir parte de los } 6,6 \text { millones de } \mathrm{m}^{2} \\
\text { que proporcionan los puertos de interés general de Vigo, } \\
\text { Marín y Vilagarcía. } \\
\text { Son innumerables las empresas que están ubicadas en } \\
\text { ZlEs (Zonas de lndustrialización espontánea repartidas por } \\
\text { toda la provincia, producto de una deficiente planificación } \\
\text { urbanística). }\end{array}$ & $\begin{array}{l}22 \text { pequeños polígonos industriales con una superficie } \\
\text { que ronda los } 4,8 \text { millones de } \mathrm{m}^{2} \text { (a la superficie anterior } \\
\text { podría añadirse parte de la superficie del puerto de Viana } \\
\text { do Castelo). } \\
\text { Son también numerosas (aunque a menor escala que en } \\
\text { Pontevedra) las empresas que están ubicadas en ZIEs } \\
\text { motivadas por una deficiente planificación urbanística. }\end{array}$ \\
\hline $\begin{array}{l}\text { Promotores } \\
\text { de poligonos } \\
\text { existentes }\end{array}$ & $\begin{array}{l}\text { Preferentemente de carácter público, esto es, Consorcio de } \\
\text { la Zona Franca de Vigo o CZFV ( } 30 \% \text { del suelo industrial } \\
\text { disponible), los ayuntamientos ( } 30 \%) \text {, el gobierno de } \\
\text { España ( } 21 \%) \text {, la Xunta de Galicia (17\%); en menor } \\
\text { medida, las instituciones privadas ( } 2 \%) \text {. }\end{array}$ & $\begin{array}{l}\text { Mayoritariamente las Cámaras Municipales ( } 60 \% \text { del suelo } \\
\text { industrial disponible), pero con una importante presencia } \\
\text { del capital privado a través de las propias asociaciones } \\
\text { empresariales ( } 33 \% \text {; por ejemplo, la sociedad Parquelnvest, } \\
\text { participada directamente por la AEP (Associação } \\
\text { Empresarial de Portugal); } 7 \% \text { otras instituciones mixtas } \\
\text { (públicas y privadas). }\end{array}$ \\
\hline $\begin{array}{l}\text { Gestores de } \\
\text { poligonos } \\
\text { existentes }\end{array}$ & $\begin{array}{l}\text { El modelo más habitual (en el } 79 \% \text { de los casos) es } \\
\text { la gestión única del ayuntamiento (infraestructuras y } \\
\text { servicios básicos que obliga la ley); entidad urbanística } \\
\text { de conservación ( } 7 \% \text { ); comunidad de propietarios ( } 7 \% \text { ); } \\
\text { el porcentaje restante ( } 7 \% \text { de los polígonos activos) es } \\
\text { realizado por otros entes públicos. }\end{array}$ & $\begin{array}{l}\text { Mayoritaria la gestión exclusiva de los ayuntamientos } \\
(63 \%) \text {; el resto entidades privadas (por ejemplo, la ya } \\
\text { comentada Parquelnvest). }\end{array}$ \\
\hline $\begin{array}{l}\text { Previsión } \\
\text { de nuevos } \\
\text { polígonos }\end{array}$ & $\begin{array}{l}29 \text { nuevos polígonos con más de } 20 \text { millones de } \mathrm{m}^{2} \text {, junto } \\
\text { con la creación de un parque tecnológico con } 350.000 \mathrm{~m}^{2} \text {; } \\
\text { horizonte } 2016 \text {. }\end{array}$ & $\begin{array}{l}15 \text { millones de } \mathrm{m}^{2} \text { repartidos en } 4 \text { plataformas logísticas y } \\
6 \text { parques industriales; horizonte estimado año } 2011 \text {. }\end{array}$ \\
\hline $\begin{array}{l}\text { Promotores } \\
\text { en los nuevos } \\
\text { Polígonos }\end{array}$ & $\begin{array}{l}19 \text { de los nuevos polígonos (65\%) promovidos por la Xunta } \\
\text { de Galicia; } 5 \text { polígonos promovidos por el CZFV }(17,24 \%) \text {; } \\
\text { el 17,24\% restante, otras entidades públicas (diputación de } \\
\text { Pontevedra y ayuntamientos) y entidades privadas. }\end{array}$ & $\begin{array}{l}\text { Se mantiene el reparto preferente municipal, con una } \\
\text { importante presencia privada. }\end{array}$ \\
\hline $\begin{array}{l}\text { Referencias } \\
\text { de precios }\end{array}$ & $\begin{array}{l}\text { Entre los } 120 \text { y los } 160 € / \mathrm{m}^{2} \text { en los nuevos polígonos } \\
\text { (en otras áreas de Galicia no de Pontevedra, entre } 12 \text { y } \\
80 € / \mathrm{m}^{2} \text { en los nuevos polígonos). }\end{array}$ & Entre los 30 y los $100 € / \mathrm{m}^{2}$ dependiendo de la zona. \\
\hline
\end{tabular}

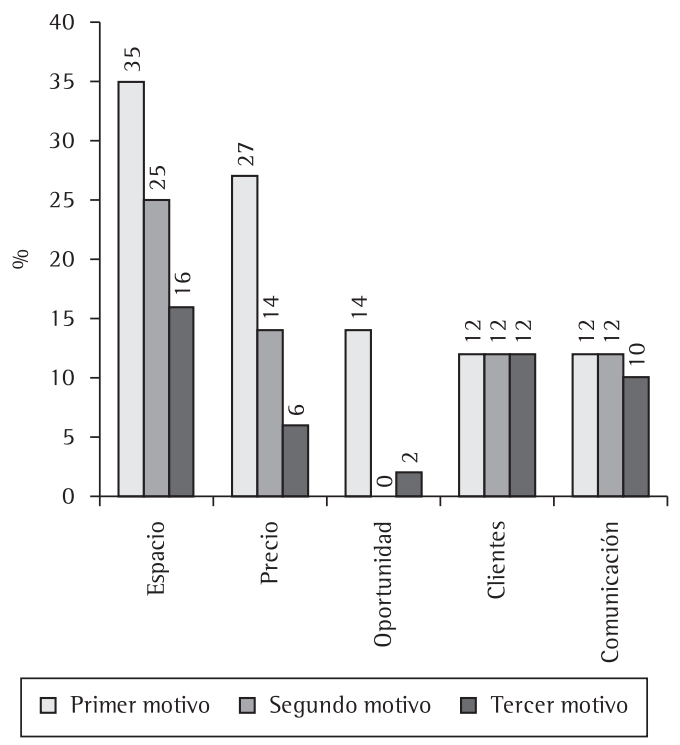

Figura 1. Principales motivos para ubicarse en el polígono actual.

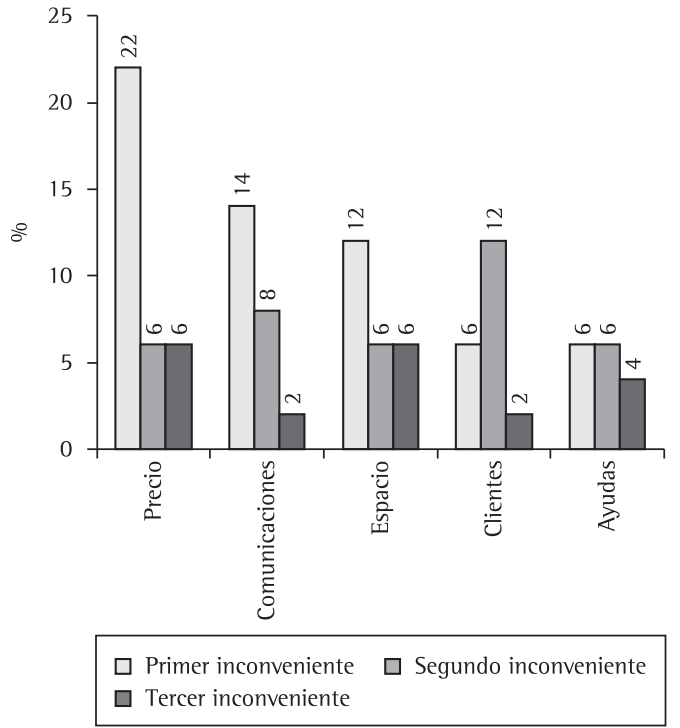

Figura 2. Principales inconvenientes encontrados para ubicarse en el polígono actual. 
la situación de su polígono. En los resultados obtenidos, se puede observar que para las empresas entrevistadas los aspectos más importantes son los accesos por carreteras de alta capacidad (4,5 en una escala 1-5 de Lykert), seguidos de la proximidad a puertos y aeropuertos (ambos con valoración de 3), mientras que la proximidad a terminales de ferrocarril tiene para las empresas una importancia secundaria $(2,25)$.

En esta misma línea, en cuanto a la satisfacción con la situación del polígono, se observa que en la localización, respecto a los accesos por carretera, las empresas están razonablemente satisfechas $(3,58)$. La satisfacción también es bastante alta para la cercanía a aeropuertos con una nota de 3,52 , sin embargo, las notas caen por debajo del tres para los otros dos aspectos (proximidad a puerto con 2,8 y terminal de ferrocarril con 2,25).

Con esta misma tipología de análisis (importancia y satisfacción) pero centrado ahora en las infraestructuras internas del polígono, se puede observar (Figura 3) que las infraestructuras a las cuales las empresas dan más importancia son, por este orden, las telecomunicaciones, el suministro eléctrico, la existencia de aparcamientos, el suministro de agua, el diseño de los viales, la señalización de los accesos, el estado de los viales y la iluminación del polígono. Como también se observa en el gráfico 3, el nivel de satisfacción es bajo (en relación con la importancia otorgada), especialmente en aspectos como los aparcamientos, el estado de los viales y las telecomunicaciones. En este último apartado, son muy frecuentes las quejas de empresas que todavía no disponen siquiera de ADSL, lo cual dificulta en gran medida su trabajo

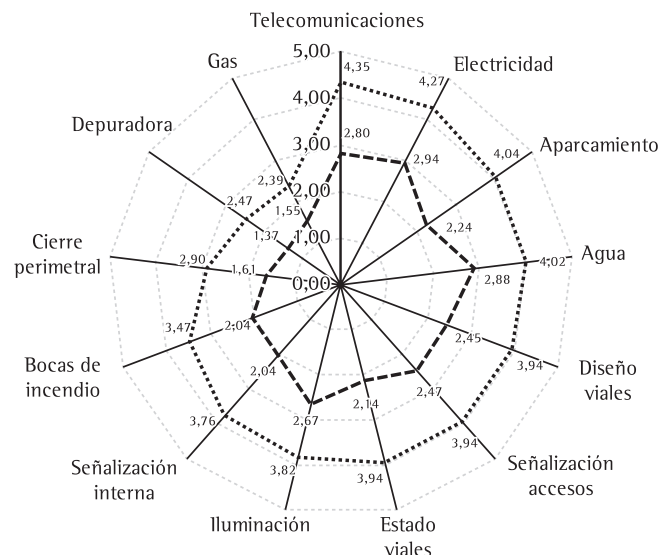

diario. Asimismo, otro aspecto a destacar es que en todos los aspectos preguntados, la satisfacción media se encuentra por debajo de la importancia.

Continuando con el raciocinio, ahora enfocado a nivel de servicios, se observa que los aspectosmás importantes para las empresas (Figura 3) son la vigilancia del polígono, recogida de residuos industriales, limpieza y recogida de residuos sólidos urbanos, atención sanitaria, respuesta ante emergencias (incluso los incendios), transporte de viajeros, servicios de restauración y existencia de guarderías. De nuevo, se observa un bajo nivel de satisfacción con casi todos los servicios mostrados, moviéndose en valores alrededor de 2, quedando de manifiesto que para vigilancia, transporte de viajeros y guardería el nivel de satisfacción es especialmente bajo. En este apartado de servicios también la satisfacción se encuentra por debajo del interés mostrado.

De forma global, sólo un 31\% de las empresas entrevistadas muestra su satisfacción Con la cobertura de sus necesidades con las infraestructuras de su polígono, el dato más negativo es en el caso de la prestación de servicios, ya que hasta un 73\% de los encuestados muestra su insatisfacción con el desarrollo actual de estos servicios en su polígono.

Direccionando el análisis hacia los promotores de los polígonos activos en Pontevedra, se resalta su baja valoración por parte de las empresas $(2,92)$, siendo sus principales quejas la carencia de infraestructuras y servicios en el momento de instalarse en las parcelas (que incluso se mantienen en la actualidad) y la lentitud en los trámites administrativos.

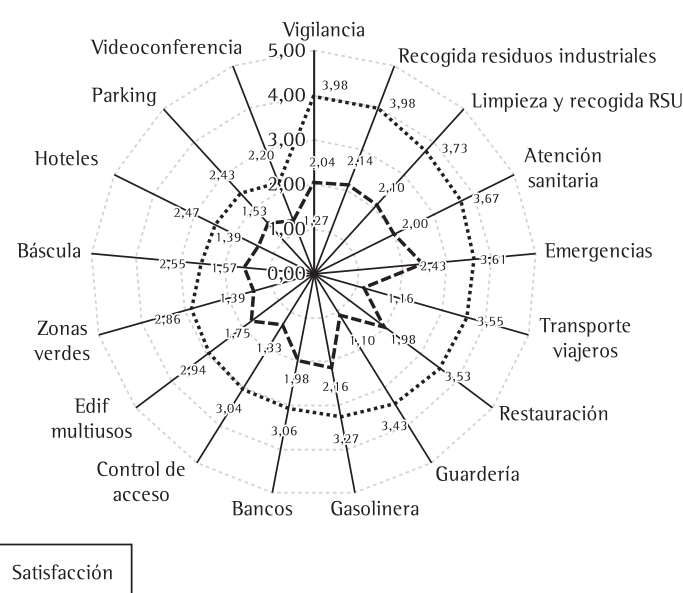

Figura 3. Valoraciones de las infraestructuras (izquierda) y servicios (derecha) del polígono. 
Cuadro 3. Recomendaciones para la mejora de la promoción y gestión de los polígonos industriales (elaboración propia).

\begin{tabular}{|c|c|}
\hline \multirow{11}{*}{ 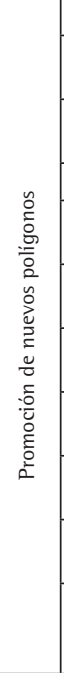 } & Cada polígono industrial debería tener, al menos, una superficie de $500.000 \mathrm{~m}^{2}$ para poder rentabilizar las inversiones en sus infraestructuras y servicios. \\
\hline & $\begin{array}{l}\text { Deberían recogerse medidas contra la especulación, contra el “feísmo" y las dedicadas a la integración del polígono en el entorno y la protección } \\
\text { medioambiental. }\end{array}$ \\
\hline & $\begin{array}{l}\text { En relación con todo las medidas anteriores deberian establecerse mecanismos de control para identificar incumplimiento de lo pactado, no sólo por } \\
\text { las empresas sino, también, por parte de promotores/concesionarias (incluyendo, mecanismos de resolución o sanción). }\end{array}$ \\
\hline & Es conveniente simultanear los procesos de urbanización y comercialización. \\
\hline & $\begin{array}{l}\text { No sólo es recomendable que el promotor pregunte a las empresas que adquieren las parcelas sus necesidades especiales sino, también, que contraste } \\
\text { el nivel de satisfacción de la empresa con su cumplimiento, previamente al inicio de la actividad. }\end{array}$ \\
\hline & $\begin{array}{l}\text { Debería mejorarse la "transparencia" en el establecimiento del precio del } \mathrm{m}^{2} \text {, de cara a proporcionar una mayor confianza de los compradores y } \\
\text { evitar situaciones especulativas. }\end{array}$ \\
\hline & $\begin{array}{l}\text { Debería reservarse espacio en los nuevos polígonos para empresas locales (especialmente si están en ZIEs), o bien, establecer criterios de preferencia } \\
\text { para las mismas. }\end{array}$ \\
\hline & $\begin{array}{l}\text { En tiempos de crisis como los actuales, seria necesario el establecimiento de fórmulas de pago y financiación flexibles, contemplando todas las } \\
\text { alternativas (compra y alquiler -con opción de compra o no-). }\end{array}$ \\
\hline & $\begin{array}{l}\text { Deberían establecerse sistemas de investigación en las administraciones, provinciales, autonómicas y nacionales que permitan establecer las } \\
\text { posibilidades competitivas de los poligonos en comparación con otras alternativas geográficas. }\end{array}$ \\
\hline & $\begin{array}{l}\text { Es necesario dimensionar, de acuerdo con la demanda, tanto el tamaño de las parcelas y de las naves como la disposición de las mismas (por ejemplo } \\
\text { en Pontevedra, naves independientes preparadas logisticamente con una superficie entre } 500 \text { y } 1.000 \mathrm{~m}^{2} \text { ). }\end{array}$ \\
\hline & $\begin{array}{l}\text { Se deberían estandarizar los procesos de planificación, desarrollo y comercialización de las parcelas y, en particular, en lo relativo a la calidad de las } \\
\text { infraestructuras y servicios, (siguiendo el ejemplo de la norma de calidad de Parquelnvest en Portugal o la propuesta de CEPE en España), de tal } \\
\text { forma que se pueda auditar y certificar la calidad de cada polígono (otro argumento comercial para salir al exterior). }\end{array}$ \\
\hline \multirow{8}{*}{ 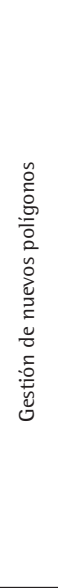 } & $\begin{array}{l}\text { Debería establecerse entidades de conservación para la gestión de los polígonos (facilita la involucración del ayuntamiento desde el inicio de su } \\
\text { actividad); además, debería establecerse claramente (deseablemente, desde el momento del planeamiento del polígono) una estimación de los costes } \\
\text { de gestión, así como la forma de financiar el ente gestor. }\end{array}$ \\
\hline & $\begin{array}{l}\text { Sería recomendable la vinculación del promotor en la gestión, de modo que se "aprendiera" de los errores y se mejoraran posteriores promociones; } \\
\text { una alternativa para esta vinculación es el mantenimiento de la titularidad de alguna(s) parcela(s). }\end{array}$ \\
\hline & $\begin{array}{l}\text { Debería potenciarse la creación de asociaciones de empresarios a la gestión de los polígonos (los nuevos y los existentes), dado que tienen mayor } \\
\text { capacidad de presión ante los organismos públicos para conseguir mejoras y, además, pueden desarrollar servicios comunes (con o sin subvenciones), } \\
\text { tales como formación, vigilancia, gestión de residuos, transporte, guardería, TICs, energía, combustible, asesoramiento jurídico y financiero,... }\end{array}$ \\
\hline & $\begin{array}{l}\text { Las asociaciones de empresarios no tendrían por qué agrupar únicamente a las empresas de un polígono, sino que podrían agrupar a empresas de } \\
\text { poligonos próximos con problemáticas similares. }\end{array}$ \\
\hline & $\begin{array}{l}\text { En el proceso de transferencia del promotor a la entidad de gestión, deberían establecerse mecanismos de coordinación entre promotor, ayuntamiento, } \\
\text { entidad de gestión y empresarios para agilizar la puesta en marcha de la gestión (por ejemplo, en el propio documento de desarrollo del polígono); } \\
\text { así, sería necesario sistematizar esta coordinación entre todos los agentes implicados, más allá de las "sintonías" personales o políticas. }\end{array}$ \\
\hline & $\begin{array}{l}\text { Sería deseable que existiese algún tipo de coordinación entre gestores de diferentes polígonos orientada a intercambiar experiencias e identificar } \\
\text { "buenas prácticas" de gestión, en línea, con las señaladas por CEPE y FEGAPE. }\end{array}$ \\
\hline & $\begin{array}{l}\text { Para optimizar los recursos dedicados a la gestión, incluso podrían estudiarse soluciones donde una entidad se encargase de gestionar o asesorar a } \\
\text { varios poligonos (modelo de Parquelnvest, por ejemplo). }\end{array}$ \\
\hline & $\begin{array}{l}\text { Deberían intensificarse los esfuerzos para mejorar la vocación de la gestión de los polígonos hacia las empresas usuarias, trabajadores y sociedad. } \\
\text { Así, desarrollos de normas ISO } 9000 \text { o ISO } 14000 \text { en los polígonos podrían facilitar este enfoque. }\end{array}$ \\
\hline \multirow{6}{*}{ 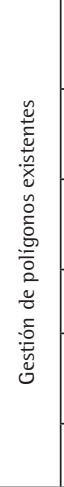 } & $\begin{array}{l}\text { En base a los comentarios de las empresas usuarias, se deberia mejorar la gestión de infraestructuras y servicios tales como suministro eléctrico, } \\
\text { limpieza y recogida de residuos, vigilancia, viales, telecomunicaciones, iluminación, bocas de incendio, recogida de residuos industriales, guarderia, } \\
\text { restauración, transporte público (puede abarcar más de un municipio) y mantenimiento de las zonas verdes. }\end{array}$ \\
\hline & $\begin{array}{l}\text { Sería conveniente constituir entidades de conservación o comunidades de propietarios en los polígonos que no dispongan de ellas. No obstante, } \\
\text { dado que éstas no se pueden crear a posteriori (salvo unanimidad de los propietarios), sería deseable que se realizasen modificaciones en la } \\
\text { legislación vigente, obligando a constituirse dichas entidades y haciendo obligatoria (o "incentivando") la pertenencia a las mismas. }\end{array}$ \\
\hline & $\begin{array}{l}\text { Sería necesaria una mayor involucración de los ayuntamientos en la gestión, realizando labores de concienciación con los mismos; además, en } \\
\text { el caso de que las acciones anteriores no diesen resultado y los ayuntamientos incumpliesen sus obligaciones sistemáticamente (incluyendo las } \\
\text { financieras), sería deseable que hubiese mecanismos de penalización, si bien, eso requeriria cambios en la legislación actual. }\end{array}$ \\
\hline & $\begin{array}{l}\text { Deberían penalizarse los problemas derivados de una deficiente prestación de servicios por parte de las empresas concesionarias (suministro eléctrico, } \\
\text { gas, agua, comunicaciones,...). }\end{array}$ \\
\hline & $\begin{array}{l}\text { Se deberían penalizar las "malas prácticas" de las empresas usuarias o de terceros, que perjudican al buen desarrollo de la actividad dentro del } \\
\text { poligono (parking abusivo, basura, ruido,...), a través de ordenanzas municipales, lo que requiere de nuevo una sensibilidad del ayuntamiento por } \\
\text { los problemas del poligono. }\end{array}$ \\
\hline & $\begin{array}{l}\text { Los problemas en los poligonos activos deberian servir para corregir deficiencias de diseño en los nuevos polígonos ("aprender de los errores"), lo } \\
\text { que demandaría foros entre promotores, gestores y asociaciones de empresarios en los que se comentasen y buscasen soluciones a estos problemas. }\end{array}$ \\
\hline \multirow{5}{*}{ 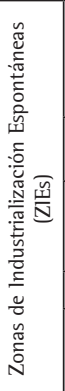 } & $\begin{array}{l}\text { Administraciones y empresas (con el apoyo de las asociaciones empresariales) deberían tomar, coordinada y rápidamente, acciones para planificar } \\
\text { el traslado o la conversión de las ZIEs (“no mirar para otro lado”); la decisión de cuál es la opción más adecuada dependiente de la normativa } \\
\text { urbanistica de la zona, la posibilidad de crear las infraestructuras adecuadas, la disponibilidad de espacio, el impacto medioambiental de las } \\
\text { empresas ubicadas en ellas, etc. }\end{array}$ \\
\hline & $\begin{array}{l}\text { En aquellos ZIEs que no puedan ser reconvertidas en polígonos, las empresas deberían contar para el cálculo global de demanda de suelo para } \\
\text { facilitar su migración. }\end{array}$ \\
\hline & $\begin{array}{l}\text { Dado que la reconversión de una ZIE en polígono industrial suele ser complejo, debería potencializarse la coordinación entre todas las partes } \\
\text { (empresas, asociaciones de empresarios, ayuntamientos -en ocasiones más de uno-, gobiernos autonómicos, etc.). Una opción interesante es la de } \\
\text { tratar de ordenar estas ZIEs mediante la elaboración de un Plan Parcial. }\end{array}$ \\
\hline & Sería interesante la creación de ayudas transitorias para facilitar esta conversión (o incluso la migración a otra ubicación). \\
\hline & $\begin{array}{l}\text { Dado que muchas de estas ZIEs afectan a varios ayuntamientos simultáneamente (con concesionarias de servicios como agua, transporte o recogida } \\
\text { de basuras diferentes) sería necesaria una mayor coordinación supramunicipal que podría resolverse, en muchos casos, mediante la creación de entes } \\
\text { coordinadores como las Áreas Metropolitanas. }\end{array}$ \\
\hline
\end{tabular}


Paralelamente, por un lado la valoración del ayuntamiento como entidad gestora es de 2,35, la cual es bastante inferior a la obtenida por las otras entidades de gestión $(3,58)$. Y por otro, es curioso que el 27\% de las empresas desconozca el papel de las entidades de gestión de su polígono, considerando que las mismas dan una respuesta adecuada a sus necesidades, sólo el 18\% de las empresas conocen su existencia.

El 57\% de las empresas considera que existe o ha existido prácticas especulativas en la concesión y asignación de las parcelas de los polígonos (sólo un $12 \%$ opina que no han existido o existen estas prácticas). Asimismo, una amplia mayoría de las empresas (el 80\%) cree que se debe luchar contra el "feísmo" y la falta de integración medioambiental de los polígonos en el entorno. No obstante, entre las empresas que creen en la necesidad de luchar contra el "feísmo" el 68\% lo haría de forma obligatoria, por un 17\% que considera estas medidas como voluntarias.

En cuanto a las necesidades futuras, casi el $40 \%$ de las empresas analizadas no puede crecer en su ubicación actual y más del 30\% necesitaría hacerlo. En esta línea, aproximadamente el 50\% de las empresas manifiesta la falta de suelo industrial en la provincia, sin embargo, sólo el 12\% de los entrevistados considera que exista falta de suelo industrial en el Norte de Portugal.

La zona fronteriza del Norte de Portugal se rige como alternativa para las empresas que buscan suelo industrial en el marco geográfico del noroeste peninsular. Esta zona portuguesa, dotada de una base empresarial tradicionalmente débil, ha ido creciendo en los últimos años gracias, entre otros aspectos, a la implantación de empresas, tanto de origen gallego como de otros ámbitos, que buscaban ubicarse en el área de influencia de Vigo con un beneficio añadido de un diferencial positivo en el coste de la mano de obra.

Así, de modo ilustrativo, los principales motivos de las 4 empresas analizadas (empresas ubicadas inicialmente en Pontevedra que, posteriormente, han trasladado/ampliado sus instalaciones a Portugal) para implantar sus naves en el Norte de Portugal coinciden con los motivos comentados por las empresas de los polígonos pontevedreses, esto, es el binomio espacio disponible a precio asequible; otros aspectos señalados son tanto la cercanía a clientes y al puerto de Vigo, como el bajo coste de la mano de obra.

En cuanto a las dificultades que se encontraron para la implantación, existen diferencias respecto a las empresas ubicadas en Pontevedra. En el
Norte de Portugal se destacan la dificultad en los trámites (incluso para acceder a subvenciones), la inexistencia de recursos humanos cualificados y las malas infraestructuras (en algunos casos, su inexistencia).

\section{Conclusiones y recomendaciones}

La escasez de suelo industrial, en condiciones adecuadas en la provincia de Pontevedra, es una deficiencia estructural crónica que se ha intensificado en los últimos años. Esto ha provocado tres efectos negativos: el aumento del precio del suelo industrial, la proliferación de empresas en ubicaciones inadecuadas (ZIEs) y la falta de atractivo de la provincia para nuevas inversiones empresariales.

Uno de los principales motivos de esta situación ha sido la deficiente planificación adoptada históricamente en materia de desarrollo de suelo industrial, que no siempre ha respondido a las necesidades económicas de las empresas ni a las posibilidades de empleo de las zonas donde se ubican (política de polígonos pequeños repartidos por toda la geografía autonómica) y cuya responsabilidad ha recaído en diferentes entes no coordinados, especialmente, de carácter público.

En esta línea, los polígonos activos de Pontevedra han estado, tradicionalmente, mal dotados a nivel de infraestructuras y servicios y, en algunos casos, mal conectados logísticamente (a juicio de sus usuarios), mermando la competitividad de las empresas, principalmente aquellas ubicadas en ZIEs, las cuales, en muchos casos, no poseen licencias provisionales de actividad, lo que impide o dificulta el acceso a subvenciones, ayudas o líneas de financiación, e incentiva también, una creciente presión social por el incumplimiento de nuevas normativas, mayoritariamente medioambientales.

Asimismo, uno de los puntos clave que ha dificultado la promoción de polígonos industriales, ha sido y es, la cantidad de municipios de la provincia que no cuentan con PGOUs aprobados (sólo el 3,2\% de los ayuntamientos de la provincia de Pontevedra tienen PGOUs aprobados); además, el proceso de desarrollo de un PGOU es complejo y largo, ayudado por el tradicional "minifundismo" del suelo gallego. Posiblemente, estos retrasos o trabas expliquen la escasa aportación de la iniciativa privada en la promoción de polígonos.

Para agilizar el desarrollo, en los nuevos polígonos se ha intensificado el uso de Proyectos 
Sectoriales (muy útiles cuando no existe PGOUs en los ayuntamientos); sorprendentemente, el empleo de los Proyectos Sectoriales estaba jurídicamente disponible desde el año 1996, si bien, sólo en los últimos años se ha extendido su uso.

Por otro lado, aún asumiendo que la identificación de necesidades de suelo industrial es un proceso complejo, raramente, se ha contado con la visión de las propias empresas, pudiendo estar este cálculo de necesidades distorsionado por el efecto de las ZIEs, la anotación de una misma empresa en varios concursos (con objeto de asegurar su ubicación en cualquier nuevo polígono) o porque estos datos no son actualizados frecuentemente (por ejemplo, la crisis actual provoca retrasos en algunas inversiones).

Incluso en la planificación de los nuevos polígonos (liderada desde la iniciativa pública) todavía persisten algunos problemas, tanto con sus infraestructuras y servicios, como con la escasa adopción de medidas para convertir/trasladar las ZIEs; más aún, un problema añadido es la lentitud en disponer de esos nuevos polígonos (sólo 7 nuevos polígonos, 36\%, estará disponible antes de 2 años).

No obstante, esta planificación futura permite vislumbrar oportunidades para nuevas inversiones (las necesidades futuras de suelo industrial en la provincia se estiman en un rango de 14-19 millones de $\mathrm{m}^{2}$ frente a los 20 millones de $\mathrm{m}^{2}$ programados (Vence, 2007)), siempre y cuando, el precio del suelo sea competitivo (sin especulación) y el polígono esté bien comunicado e integrado en el entorno.

En otro sentido, los polígonos existentes cuentan con una gestión casi exclusivamente municipal, este modelo, con el que las empresas están claramente insatisfechas, se produce porque ni en la legislación urbanística ni en los proyectos de desarrollo de los polígonos se consideró otro modelo de gestión. Los problemas con los ayuntamientos en polígonos nuevos o activos arrancan desde el mismo proceso de transferencia del promotor, dado que no se ha cuidado especialmente esta coordinación entre las diferentes entes participantes. En general y desde la perspectiva de las empresas usuarias, los polígonos no sólo arrancan con una serie de problemas en sus infraestructuras y servicios, sino, en muchos casos, estos problemas persisten en el tiempo (especialmente, si la gestión es exclusiva del ayuntamiento). Por otro lado, las ZIEs tienen que ser replanteadas, ya sea porque las empresas las abandonen y se trasladen a polígonos industriales, o bien porque estas ZlEs se reconviertan en verdaderos polígonos.
La disponibilidad de suelo industrial a precios competitivos en el Norte de Portugal junto con las mejoras de sus infraestructuras logísticas con Galicia y el atractivo de su coste de mano de obra, constituyen una amenaza real de deslocalización de las empresas gallegas hacia el Norte de Portugal (GARCÍA-ARCA; PRADO-PRADO, 2007). No obstante, esta disponibilidad de $\mathrm{m}^{2}$ en Portugal también debería ser contemplada como una oportunidad de atraer nuevas inversiones a la Eurorregión que redunde en oportunidades de negocio. En Portugal, a diferencia de Galicia y España sí han sabido profesionalizar y no ser totalmente dependiente del ente público para la promoción y gestión de polígonos, desarrollando entidades (como Parquelnvest) que crean sociedades para gestionar cada polígono y a las que cede el "know-how" necesario para hacerlo correctamente (incluyendo el desarrollo de nuevos servicios adaptados a las necesidades empresariales e, incluso, compartiendo recursos entre polígonos).

A modo de conclusiones los autores proporcionan una serie de recomendaciones fruto, no sólo de la revisión bibliográfica sino, especialmente, del contraste de opiniones y reflexiones con los diferentes colectivos analizados y que son extrapolables al ámbito de la península ibérica.

Así, en la promoción de nuevos polígonos, los gobiernos autonómicos deberían desarrollar un "mapa de demanda" de suelo industrial para un período determinado y con una visión geográfica amplia que incluya no sólo la demanda de nuevas empresas sino, también, las necesidades de suelo para las ampliaciones de empresas ya existentes, incluyendo el traslado de empresas ubicadas en ZIEs. Esta identificación de la demanda debería realizarse conjuntamente entre las administraciones (autonómicas y locales) y las empresas o sectores de la zona, a través de las correspondientes asociaciones empresariales.

En este contexto, áreas, comunidades y países limítrofes no deberían contemplarse sólo como zonas competidoras sino, para algunos sectores e industrias, complementarias (RAVELO, 2002; ULGAD0, 1996). Esto debería suponer la potenciación de la coordinación entre administraciones y empresas sistematizando, por ejemplo, foros donde se compartan problemas y soluciones relacionadas con la promoción y gestión de los polígonos. Con todo ello, se lograría que la creación de polígonos se pudiera realizar con antelación suficiente, de modo que existiese siempre un cierto excedente de suelo, procurando que sea "el suelo el que espere por las empresas y no al contrario"; evidentemente, esta anticipación debería extenderse a la disponibilidad 
de infraestructuras logísticas que comunican al polígono industrial.

Además, los datos de demanda deberían actualizarse periódicamente, siendo recomendable que existiese un único registro de demanda de $\mathrm{m}^{2}$ demandados por las empresas a nivel autonómico (para evitar duplicidades). Es evidente que la selección de las zonas dónde crear los polígonos y su superficie debería basarse, fundamentalmente, en criterios de demanda empresarial, con mínimas condiciones del entorno (mano de obra cualificada, desarrollo de servicios para las empresas y los trabajadores,...) y buenas comunicaciones logísticas. Históricamente en Pontevedra y en el Norte de Portugal los criterios de ubicación no han sido suficientemente objetivos con lo que no se ha respondido a las necesidades empresariales.

Por otro lado, el desarrollo de un nuevo polígono industrial debería partir de los PGOUs (una forma de involucrar a los ayuntamientos en su gestión), por lo que debería acelerase el proceso de desarrollo de estos PGOUs, dotando de mayores recursos a los ayuntamientos y con una perspectiva coordinada entre ayuntamientos limítrofes. La alternativa de los Proyectos Sectoriales se ha mostrado como una herramienta eficaz para el desarrollo de suelo industrial en una situación de escasez de suelo industrial y de ausencia habitual de PGOUs, si bien, presenta el inconveniente de haberse realizado sin la necesaria coordinación con los ayuntamientos afectados (responsables o co-responsables de la posterior gestión del polígono).

Asimismo, previamente a la comercialización, debería establecerse un compromiso de ejecución de infraestructuras logísticas, infraestructuras y servicios del polígono por parte del promotor (con plazo y presupuesto); de hecho, a todas las administraciones y entes privados se les debería exigir, mayor rigor y seriedad en los plazos de ejecución de los polígonos. De este modo, previamente a la urbanización del polígono industrial, se podría realizar una estimación fiable y realista del coste del $\mathrm{m}^{2}$ de suelo urbanizado en el polígono (para evitar sorpresas de financiación a las empresas cuando paguen sus parcelas).

lgualmente, deberían especificarse claramente: la situación y las dimensiones de las parcelas, los viales, la iluminación, el tipo de cierre perimetral, las zonas de parking, las conexiones logísticas, la reserva de $\mathrm{m}^{2}$ para futuras ampliaciones del polígono, la tipología de las redes de agua, electricidad y gas, así como la del sistema "contraincendios" y de telecomunicaciones, la existencia de zonas verdes y áreas de restauración, comercio, etc. Paralelamente, también, debería especificarse el tipo de entidad de gestión que se hará cargo del mantenimiento del polígono (preferentemente, entidades de conservación), la previsión de generación de servicios una vez que arranque la actividad en el polígono (transporte de pasajeros, guardería, gestión de residuos industriales,...).

A medida que se vayan ajustando la oferta $y$ demanda de suelo industrial, debería tenderse a una cierta especialización de los polígonos industriales (sectoriales, tecnológicos,...) para racionalizar las infraestructuras y los servicios ofertados; así, en los casos que exista excedente de suelo se deberian elaborar programas de comunicación conjuntos y coordinados en el exterior orientados a la búsqueda de nuevas inversiones o empresas que puedan ubicarse en la Eurorregión (si el precio final no es atractivo, la otra alternativa es vender la calidad de sus infraestructuras y servicios).

Finalmente, de forma más resumida (Cuadro 3), se recogen otras recomendaciones asociadas, no sólo a la promoción de polígonos sino, también, a su gestión (tanto en los nuevos polígonos como en los polígonos ya en funcionamiento). Asimismo, se incluyen una serie de recomendaciones para mejorar la situación de las ZIEs.

\section{Referencias}

CHRISTOPHER, M.; TOWILL, D. R. Developing market specific supply chain strategies. The International Journal of Logistics Management, v. 13, n. 1, p. 1-14, 2002. http:// dx.doi.org/10.1108/09574090210806324

FEDERACIÓN DE POLÍGONOS EMPRESARIALES DE LA COMUNIDAD VALENCIANA - FEVEPAL. Manual para la creación y gestión de entidades gestoras de áreas empresariales. Valencia: FEPEVAL, 2006.

FEDERACIÓN GALLEGA DE PARQUES EMPRESARIALES - FEGAPE. Guía de boas prácticas para a xestión dos parques empresariais en Galiza. Santiago de Compostela: FEGAPE, 2008.

GARCÍA-ARCA, J.; PRADO-PRADO, J. C. Diagnose e plan de actuacións de mellora para a loxística nos sectores alimentario e textil-moda de Galicia. In: SEMINARIO DE LOXíSTICA, 12., 2007, Santiago de Compostela. Anais...

GARCÍA-CEBRIÁN, L.; MUÑOZ, A. Análisis de los resultados obtenidos por la aplicación de diversas metodologías de ayuda a la decisión multicriterio. Consistencia de los resultados obtenidos. In: CONGRESO INTERNACIONAL DE INGENIERÍA DE ORGANIZACIÓN, 4., 2009, Barcelona. Anais...

KEARNEY, A. T. Excellence in logistics. Chicago, 2009.

MORA, F. Nuevas formas de gestión de las áreas empresariales. Asturias: CEPE, 2006.

RAVELO, T. et al. La localización industrial en Canarias. Una aproximación multicriterio. Las Palmas: Fundación FYDE-Caja Canarias, 2002. 
ULGADO, F. Location characteristics of manufacturing investments in the United States: a comparation of American and Foreign-based firms. Management International Review, v. 36, n. 1, p. 7-26, 1996.
VENCE, X. As necesidades empresariais de solo produtivo en Galicia 2006-2015 e programa de actuacións estratéxicas en solos produtivos. Santiago de Compostela: Consellería de Vivenda e Solo e Consellería de Innovación e Industria, 2007.

\title{
Promotion and management in industrial states of the Euroregion Galicia-North of Portugal
}

\begin{abstract}
This paper not only makes a deep analysis of the main causes that imply problems in promotion and management in industrial estates, but also proposes an action plan to improve the current situation. In order to illustrate these problems, the situation of industrial estates in the Euroregion Galicia-North of Portugal (Pontevedra area in Spain and Minho-Lima area in Viana do Castelo, Portugal) was presented. Although the study focused only on these geographical areas ( 55 companies), the conclusions and recommendations could be easily extrapolated to the rest of Spain and Portugal. Among these conclusions, the need for a better strategy to locate new industrial estates, for a higher development of services and infrastructures, and for the adoption of suitable organizational schemes to the maintenance of industrial estates, could be outlined.
\end{abstract}

\section{Keywords}

Industrial estates. Promotion. Management. 